
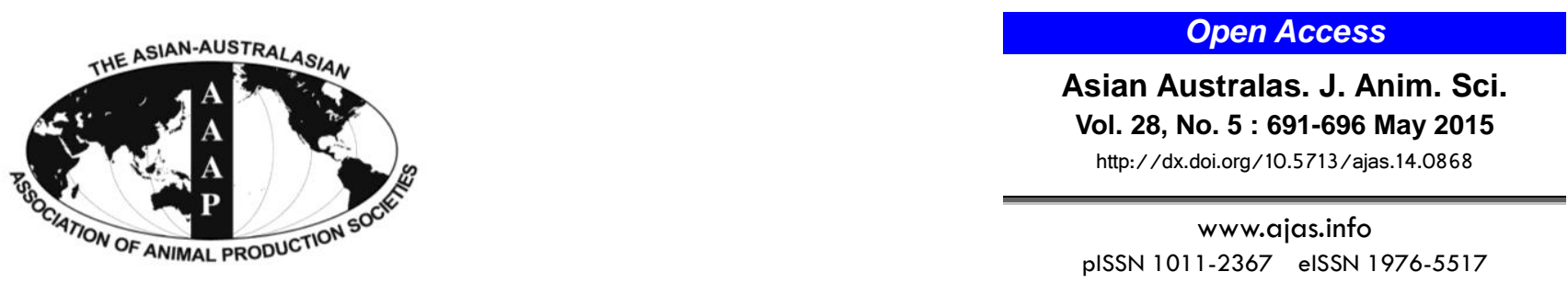

\title{
Quality Evaluation of Five Commercial Enzyme Linked Immunosorbent Assay Kits for Detecting Aflatoxin $B_{1}$ in Feedstuffs
}

\author{
Dan-dan Sun, Xu Gu, Jun-guo Li, Ting Yao, and Ying-chao Dong* \\ Feed Research Institute, Chinese Academy of Agricultural Sciences, Beijing 100081, China
}

\begin{abstract}
The objective of this study was to evaluate the quality of five commercial enzyme linked immunosorbent assay (ELISA) kits $(\mathrm{A}, \mathrm{B}, \mathrm{C}, \mathrm{D}$, and $\mathrm{E})$ from different suppliers for detecting aflatoxin $\mathrm{B}_{1}\left(\mathrm{AFB}_{1}\right)$. $\mathrm{AFB}_{1}$-free corn samples supplemented with different levels of $\mathrm{AFB}_{1}(5,10$, and $20 \mu \mathrm{g} / \mathrm{kg}$ ) were used as positive controls and 6 replicates of each control sample were tested to evaluate the accuracy and precision of these kits. In addition, we also evaluated the performance of these ELISA kits for AFB ${ }_{1}$ in 30 feed samples, including corn, distillers dried grains with soluble, wheat samples, soybean meal, and poultry feed, which were verified by high performance liquid chromatography. Results showed that the coefficients of variation ranged from $1.18 \%$ to $16.22 \%$ in intra-plate and $2.85 \%$ to $18.04 \%$ in inter-plate for the determination of $\mathrm{AFB}_{1}$. The half maximal inhibitory concentration for five kits ranged from 3.72 to $7.22 \mu \mathrm{g} / \mathrm{kg}$. The quantitation limits of $\mathrm{AFB}_{1}$ were all under the legal limit in China but somewhat inconsistent with kit instructions. Although the recovery rate of four of the five kits were either less than $90 \%$ or more than $110 \%$, all these values were acceptable in practice. Two kits had high false positive rates (C and E). In conclusion, our results revealed that the qualities of five tested ELISA kits were significantly different. (Key Words: Enzyme Linked Immunosorbent Assay Kit, Aflatoxin $B_{1}$, Evaluation, Feedstuffs)
\end{abstract}

\section{INTRODUCTION}

Aflatoxins (AFs) are potent, natural toxins produced by Aspergillus flavus, Aspergillus nomius, or Aspergillus parasiticus (Sweeney et al., 1998), which frequently contaminate foods and animal feedstuffs (Guan et al., 2011). In particular, feeds with high concentrations of plant materials, such as peanut, corn, soybean and rice, are more susceptible to mycotoxin contamination (Zychowski et al., 2013). Aflatoxins have caused a significant problem for the animal feed industry and been an ongoing risk to feed supply security (Bryden, 2012). AFs are of great concern because of their detrimental effects on the health of human and animals such as carcinogenic, mutagenic, teratogenic, and immunosuppressive effects (Toteja et al., 2006; Zinedine et al., 2012).

\footnotetext{
* Corresponding Author: Ying-chao Dong. Tel: +86-82106069, E-mail: dongyingchao@caas.cn

Submitted Nov. 11, 2014; Revised Dec. 30, 2014; Accepted Jan. 15, 2015
}

Aflatoxin $\mathrm{B}_{1}\left(\mathrm{AFB}_{1}\right)$, one of the most common toxins in foods and feeds, has been classified as a group 1 carcinogen by the International Agency for Research on Cancer of World Health Organization (IARC, 1993). It is harmful to human and animals because of its diverse toxicity that causes weight loss, immunosuppression, mutagenesis, reproductive alterations and carcinogenesis (Zychowski et al., 2013). Studies have showed that the prolonged feeding of gibel carp with high levels of $\mathrm{AFB}_{1}$ can inhibit reproduction activity by reducing fecundity and egg size (Huang et al., 2014); low level mycotoxin in swine and poultry diets can result in the reduction of feed intake, growth rate, egg production, carcass quality, fertility, hatchability of eggs, and immunosuppression (Harvey et al., 1991; Bryden, 2012; He et al., 2013). The ruminants fed with $\mathrm{AFB}_{1}$ contaminated feed can transfer $\mathrm{AFB}_{1}$ into milk in the form of $A F s M_{1}$, which is classified as a possible human carcinogen (group 2B) (IARC, 1993).

In addition, the widespread distribution of $\mathrm{AFB}_{1}$ could be another reason for being a hazard (Binder et al., 2007). 
$\mathrm{AFB}_{1}$ can be found in a wide range of feedstuffs, depending on the regional differences and climate prevailing on the harvest site (Pleadin et al., 2014). It is not only the main testing items of feed hygiene standards but also the monitoring object of mycotoxins occurrence in feed and raw materials worldwide. Many surveys have showed the status of $\mathrm{AFB}_{1}$ concentrations in feedstuffs around the world (Binder et al., 2007; Streit et al., 2012; Anukul et al., 2013; Streit et al., 2013; Zachariasova et al., 2014).

Due to the frequent occurrence and toxicity of $\mathrm{AFB}_{1}$, many countries and regions have set maximum limits for $\mathrm{AFB}_{1}$. A survey shows that approximately 100 countries have developed specific limits for mycotoxins in food and feedstuffs and all countries with mycotoxin regulations have at least regulatory limits for $\mathrm{AFB}_{1}$ or the sum of $\mathrm{AFs}$ $B_{1}, B_{2}, G_{1}$, and $G_{2}$ in foods and/or feeds (Binder, 2007). For example, a legal limit for $\mathrm{AFB}_{1}$ in corn for animals is 50 $\mu \mathrm{g} / \mathrm{kg}$ in China, Japan and Korea; the U.S. and Canada have set the legal limit of $20 \mathrm{mg} / \mathrm{kg}$ for total $\mathrm{AFs}\left(\mathrm{AFB}_{1}+\mathrm{AFB}_{2}+\right.$ $\mathrm{AFG}_{1}+\mathrm{AFG}_{2}$ ) (GB 13078-2001; Van Egmond, 2004). Details of worldwide regulations for $\mathrm{AFB}_{1}$ in food and feed are listed in a review (Van Egmond, 2004).

The low permissible limits of $\mathrm{AFB}_{1}$ require rapid, sensitive and specific analytical methods to quantify trace levels for quality control and safety assessment of feeds (Guo et al., 2014; Xie et al., 2014). Availability of immunochemical method has led to the development of many rapid and sensitive methods for monitoring and quantifying $\mathrm{AFB}_{1}$ in contaminated foods and feeds (Liu et al., 2013). Enzyme linked immunosorbent assay (ELISA) is a convenient screening tool which can provide a great saving in cost, resources, efforts and toxic solvents (Zhang et al., 2011). This method has been shown to be simple, portable, and reliable for screening a large number of samples (Li et al., 2009; Guan et al., 2011), and had become the most common rapid method for mycotoxin detection in foods and feeds (Zheng et al., 2005; Wang et al., 2011). Additionally, the detection limits of ELISA can be comparable with or even lower than those obtained by instrumental methods (Zhang et al., 2011). However, differences exist in commercial ELISA kits for the detection of $\mathrm{AFB}_{1}$. Some manufactures only launches one ELISA product for detecting $\mathrm{AFB}_{1}$ in all substance, which may reduce the performance of ELISA kits because different inferring substances co-extract from different food and feed matrixes (Li et al., 2009). Furthermore, a number of factors, such as antibodies, co-extracted compounds, the power hydrogen of the extract, and the composition of the extraction solvent, can result in over- or underestimation of $\mathrm{AFB}_{1}$ contamination. As a result, the $\mathrm{AFB}_{1}$ concentration of a same sample might be discrepant by kits from different manufacturers and even different batches of the same manufacturers. As commercialized ELISA kits are very important for monitoring $\mathrm{AFB}_{1}$ in feeds, an extensive study on the accuracy and reproducibility of different commercial ELISA kits is needed. The purpose of the present study was to evaluate the performance of five commercialized ELISA test kits for $\mathrm{AFB}_{1}$ in feed and recommend customers to keep monitoring $\mathrm{AFB}_{1}$ kits during using.

\section{MATERIALS AND METHODS}

\section{Samples}

A total of 30 feed samples that are natural contaminated by $\mathrm{AFB}_{1}$, including corn (5), distillers dried grains with soluble (5), wheat samples (5), soybean meal (5), and 10 poultry feed ( 5 for layer and 5 for broiler), were obtained from feed companies in China. Three batches of $\mathrm{AFB}_{1}$ ELISA kits were collected from each company. The prepared test samples were ground into a fine powder with a particle size of $1.0 \mathrm{~mm}$ using an analytical mill (Retsch ZM100; Haan, Germany) and stored at $4^{\circ} \mathrm{C}$ prior to $\mathrm{AFB}_{1}$ analysis.

\section{Enzyme linked immunosorbent assay method}

Five commercially available ELISA kits from Beijing Kwinbon Biotechnology Co., Ltd., China; Huaan Magnech Biotechnology Co., Ltd. China; Beijing Longkefangzhou Biotechnology Co., Ltd. China; ROMER International Trade (Beijing) Co., Ltd., China; and ADWK Biotechnology Co., Ltd. Beijing, China, were randomly assigned to A, B, C, D, and E. All results are consistently presented using these letters. Four of these kits were produced by domestic companies while the other one was from a foreign company. The assays were conducted following manufacturers' instructions.

The evaluated parameters included linearity, sensitivity, accuracy, and precision. The linearity was assessed by the linear regression analysis and five levels of standard solutions in duplicates, according to respective kit instructions, were used to establish the standard curves for each plate. Commonly used methods to evaluate kit sensitivity are limit of detection (LOD) and the half maximal inhibitory concentration (IC50). The LOD was calculated from the mean value obtained with eighteen blank corn samples plus three standard deviations and the limit of quantification (LOQ) was the mean value plus ten standard deviations. The IC50, the concentration of $\mathrm{AFB}_{1}$ at $50 \%$ absorbance of zero standard solution, could be calculated from standard curve. Accuracy and precision were based on the aflatoxin recovery tests. Feed samples intended for corn with non-detectable $\mathrm{AFB}_{1}$ levels by high performance liquid chromatography (HPLC) were spiked with $\mathrm{AFB}_{1}$ at levels of 5,10, and $20 \mu \mathrm{g} / \mathrm{kg}$ and then worked out recovery rate for each level. The precision of the method was determined by the coefficient of variation $(\mathrm{CV})$ 
Table 1. CV of intra-plate and inter-plate for the five kits

\begin{tabular}{lcccccc}
\hline & $\mathrm{AFB}_{1}$ spiking level & \multicolumn{5}{c}{$\mathrm{CV}(\%)$} \\
\cline { 3 - 7 }$(\mu \mathrm{g} / \mathrm{kg})$ & Kit A & Kit B & Kit C & Kit D & Kit E \\
\hline Intra-plate & 5 & 8.93 & 9.85 & 16.22 & 9.05 & 10.95 \\
& 10 & 10.02 & 8.35 & 5.99 & 1.18 & 7.40 \\
& 20 & 7.85 & 1.73 & 10.04 & 3.83 & 9.61 \\
Inter-plate & 5 & 11.91 & 9.52 & 5.85 & 2.85 & 18.04 \\
& 10 & 6.88 & 11.77 & 6.08 & 3.55 & 8.46 \\
& 20 & 6.95 & 6.96 & 4.19 & 7.83 & 4.48 \\
\hline
\end{tabular}

$\overline{\mathrm{CV}}$, coefficient of variation; AFB1, aflatoxin $\mathrm{B}_{1}$.

of intra-plate and inter-batch. The CV of intra-plate was the ratio of the standard deviation to the mean of six parallel micro wells in a same plate at each $\mathrm{AFB}_{1}$ level and the $\mathrm{CV}$ of inter- plate was the ratio of the standard deviation to the mean of three plates at each $\mathrm{AFB}_{1}$ level. At the same time, thirty real feed samples that the $\mathrm{AFB}_{1}$ concentrations were determined by HPLC method were used to validate the accuracy of these kits. The absorbance was measured at 450 $\mathrm{nm}$ in an ELISA microplate reader (power wave XS2; BioTek Instruments, Burlington, Vermont, USA).

\section{High performance liquid chromatography method}

Extraction and clean-up: Aflatoxin extraction and clean-up were carried out using $\mathrm{AFB}_{1}$ Test immuno-affinity columns (Huaan Magnech Bio-Tech Co., Ltd, China), according to manufacturer's instructions for feed samples. Five grams of each feed sample were added with $1 \mathrm{~g} \mathrm{NaCl}$ and $25 \mathrm{~mL}$ methanol: water (70:30, v/v). The mixture was swirled for $3 \mathrm{~min}$ on the high speed homogenizer (VortexQilinbeier 2; Scientific Industries, INC, New York, USA) and afterwards filtrated. Then, $10 \mathrm{~mL}$ filtrate was diluted with $20 \mathrm{~mL}$ ultrapure water, mixed and filtrated through micro fiber filter (Huaan Magnech Bio-Tech Co., Ltd, China). This was followed by passing $15 \mathrm{~mL}$ filtrate through immune affinity column. The column was eluted by $1 \mathrm{~mL}$ methanol and then the filtrate used for the liquid chromatography (LC) system.

HPLC analysis: A Shimadzu (LC-15C; Kyoto, Japan) LC equipped with two pumps and a RF-20A fluorescence detector were used. LC separation was performed on SBC18 columns $(150 \times 4.6,5 \mu \mathrm{m}$; Agilent, Santa Clara, CA, USA) at the flow rate of $1 \mathrm{~mL} / \mathrm{min}$ and the temperature of $30^{\circ} \mathrm{C}$. The mobile phase consisted of methanol and water (40/60, V/V) and the injection volume was $15 \mu \mathrm{L}$. Detection of $\mathrm{AFB}_{1}$ was carried out using 365 and $435 \mathrm{~nm}$ as wavelengths for excitation and emission, respectively. Peak areas were used for quantification.

\section{RESULTS AND DISCUSSION}

\section{Precision}

The CVs of $\mathrm{AFB}_{1}$ from five commercial kits are shown in Table 1. All CVs of intra-plate were equal or less than $10 \%$ except for kit C and E (16.22\% and $10.95 \%$ respectively). The $\mathrm{CV}$ s of inter-batch showed that kit $\mathrm{C}$ and $\mathrm{D}$ had the best stability while kit $\mathrm{A}$ and $\mathrm{E}$ were inferior at 5 $\mu \mathrm{g} / \mathrm{kg}$ spiked level and kit B at $10 \mu \mathrm{g} / \mathrm{kg}$. The precision marked on instructions of kit $\mathrm{A}$ and $\mathrm{C}$ was less than $10 \%$ in intra-plate and inter-plate, kit D was less than $5 \%$ in intraplate and $10 \%$ in inter-plate, kit $\mathrm{E}$ was less than $10 \%$ in intra-plate and $15 \%$ in inter-plate and kit B unmarked the precision on the instruction. So the data suggested that the precision of ELISA kits for $\mathrm{AFB}_{1}$ had deviation with the values marked on the instructions. However, the precision of national standard for detecting $\mathrm{AFB}_{1}$ in animal feeding should not exceed 10\% (GB/T 17480-2008).

\section{Sensitivity}

The LOD and IC50 were used to evaluate the sensitivity of ELISA kit. As shown in Table 2, kit E had the lowest LOD and kit B had the lowest LOQ. The LOD marked on instructions were inconsistent with our data, which might be due to different plates with tiny diversity. Another reason could be the way we calculated LOD. In the present study, LOD was calculated from the average value obtained with eighteen blank samples plus three standard deviations. The way to determined LOD could be different. For example, others used the mean (plus 2 standard deviations) of ten blank samples (Zheng et al., 2005), a threefold confidence interval of zero analyte dose signal (Zhang, 2011) or other ways. Although the LOQ of five kits were quite different, all met the requirements of $\mathrm{AFB}_{1}$ detection in feeds that the most stringent regulation is less than $10 \mu \mathrm{g} / \mathrm{kg}$ in China (Guo et al., 2014). Early studies showed that LOD of AFB $_{1}$ was $1 \mu \mathrm{g} / \mathrm{kg}$ in maize using direct competitive ELISA (Karami-Osbooet al., 2012). Rossi (Rossi et al., 2012)

Table 2. LOD, LOQ, and LOD marked in instructions (LODM) for five kits

\begin{tabular}{lccccc}
\hline & Kit A & Kit B & Kit C & Kit D & Kit E \\
\hline LOD $(\mu \mathrm{g} / \mathrm{kg})$ & 1.48 & 0.74 & 2.33 & 3.41 & 0.60 \\
LOQ $(\mu \mathrm{g} / \mathrm{kg})$ & 6.00 & 3.09 & 5.38 & 5.51 & 4.29 \\
LODM $(\mu \mathrm{g} / \mathrm{kg})$ & 1.25 & 1 & 3 & 5 & 2 \\
\hline
\end{tabular}


Table 3. Average values of IC50 and CV for five kits

\begin{tabular}{lccccc}
\hline & Kit A & Kit B & Kit C & Kit D & Kit E \\
\hline IC50 $(\mu \mathrm{g} / \mathrm{kg})$ & 5.44 & 5.32 & 4.43 & 3.72 & 7.22 \\
$\mathrm{CV}(\%)$ & 10.80 & 7.78 & 4.49 & 1.14 & 9.05 \\
\hline
\end{tabular}

IC50, half maximal inhibitory concentration; $\mathrm{CV}$, coefficient of variation.

reported that LOQs in broiler feed and laying hen feed were 1.43 and $1.75 \mu \mathrm{g} / \mathrm{kg}$ using indirect competitive ELISA (icELISA) based on an anti- $\mathrm{AFB}_{1}$ monoclonal antibody, respectively. The ELISA instructions indicated that A, B, and $\mathrm{E}$ used ic-ELISA while $\mathrm{C}$ and $\mathrm{E}$ used direct competitive ELISA. For the LODs of five kits, our data support the view that ci-ELISA is more sensitive than the competitive direct ELISA (Zhang et al., 2011). Matrix materials also had great effects on results because of the different inferring substances co-extracted from different food and feed matrixes (Lee et al., 2004). In this study, four of the five kits apply to cereals, grains as raw material in food and feed and meat while one (kit C) is exclusively used in feed. These results demonstrated that antibody type, reaction type, and matrix might have effects on the ELISA test kits for LOQ or LOD. All these factors may explain variances in sensitivity of these kits.

IC50 was another index for sensitivity, which was calculated through the standard curve. The IC50 was the average of three batches ranged from 3.72 to $7.22 \mu \mathrm{g} / \mathrm{kg}$ and kit D had the lowest value (Table 3). The CVs of IC50 of all kits were different, which might reflect the difference of inter-plates of five kits.

\section{Accuracy}

A recovery study was performed out to test the accuracy of the five ELISA kits for the determination of $\mathrm{AFB}_{1}$ in $\mathrm{AFB}_{1}$-free corn samples and samples spiked with different concentration of $\mathrm{AFB}_{1}(5,10$, and $20 \mu \mathrm{g} / \mathrm{kg})$. Excellent recovery rates were found for kit $\mathrm{A}$ from fortified samples at the level of 5 to $20 \mu \mathrm{g} / \mathrm{kg}$, ranging from $85.50 \%$ to $93.92 \%$ (Table 4), which were much closer to $100 \%$ than any other kits. Compared with kit $\mathrm{A}$, kit $\mathrm{C}$ has a lower recovery rates at $5 \mu \mathrm{g} / \mathrm{kg}$. Literatures showed that the recoveries of spiked rice samples with 10 to $500 \mu \mathrm{g} / \mathrm{kg} \mathrm{AFB}_{1}$ were from $94 \%$ to $113 \%$ using direct competitive ELISA using a monoclonal antibody (Kolosova et al., 2006). According to the Commission of the European Communities (2006), the critical values for recovery rates of $\mathrm{AFB}_{1}$ are $70 \%$ to $110 \%$ for concentrations between 1 and $10 \mu \mathrm{g} / \mathrm{kg}$ and $80 \%$ to
$110 \%$ for concentrations higher than $10 \mu \mathrm{g} / \mathrm{kg}$. Therefore, only kit $\mathrm{A}$ and $\mathrm{C}$ met the recommended standards. Although the recovery rates of other kits were either less than $80 \%$ or more than $110 \%$, all the values were acceptable in practice.

\section{Linearity}

The linearity was assessed according to the linear regression analysis with five levels of standard solutions in duplicate. Standard curve was drawn using a mathematical model of $\log ($ dose $)-\operatorname{logit}\left(\mathrm{B} / \mathrm{B}_{0}\right)$ :

$$
\operatorname{logit}\left(\mathrm{B} / \mathrm{B}_{0}\right)=\ln \frac{\mathrm{B} / \mathrm{B}_{0}}{1-\mathrm{B} / \mathrm{B}_{0}}
$$

The standard curves of kit $\mathrm{A}, \mathrm{B}$, and $\mathrm{E}$ were linear relationships while that of kit $C$ was curvilinear (Table 5). It is generally believed that linear relationship is better than curvilinear relationship.

\section{Analysis of feed samples}

The concentrations of 30 feed samples for $\mathrm{AFB}_{1}$ were determined by ELISA kits and HPLC was used as a reference method. The calibration curve of HPLC method for $\mathrm{AFB}_{1}$ was constructed with standards of $0,0.625,1.25$, $2.5,5$, and $10 \mu \mathrm{g} / \mathrm{kg}$ with the LOD of $0.15 \mu \mathrm{g} / \mathrm{kg}$ and LOQ of $0.5 \mu \mathrm{g} / \mathrm{kg}$. The correlation coefficient of standard curve was 0.992. The retention time of $\mathrm{AFB}_{1}$ was about $9.9 \mathrm{~min}$. The average recovery rate was $108.7 \%$ at $10 \mu \mathrm{g} / \mathrm{kg} \mathrm{AFB}_{1}$ spiking in corn samples and $104.3 \%$ at $20 \mu \mathrm{g} / \mathrm{kg}$.

The abilities to detect $\mathrm{AFB}_{1}$ of five commercial kits were evaluated by feed samples in current study. The $\mathrm{AFB}_{1^{-}}$ concentrations of 30 feed samples were under $0.5 \mu \mathrm{g} / \mathrm{kg}$ except for two samples $(3.2 \mu \mathrm{g} / \mathrm{kg}$ and $13.5 \mu \mathrm{g} / \mathrm{kg}$, respectively), which could not be representative in the linear range of ELISA kits. However, the results reflected the abilities of screening samples that concentrations of $\mathrm{AFB}_{1}$ were below LODs to some extent. Therefore, we selected respective LODs marked on instructions as judgment standards. Our results shown in Table 6 indicated that the five commercial kits exhibited great differences in accuracy, and kit A and D performed better than others and

Table 5. Comparison of linear relationships for five kits

\begin{tabular}{lccccc}
\hline & Kit A & Kit B & Kit C & Kit D & Kit E \\
\hline $\begin{array}{l}\text { Linear } \\
\text { relationships }\end{array}$ & 0.9968 & 0.9983 & Curve & 0.9843 & 0.9922 \\
& & & fitting & & \\
\hline
\end{tabular}

Table 4. Recovery rates for five kits

\begin{tabular}{llclll}
\hline $\begin{array}{l}\text { Aflatoxin } B_{1} \text { spiking level } \\
(\mu \mathrm{g} / \mathrm{kg})\end{array}$ & Kit A & Kit B & Kit C & Kit D & Kit E \\
\hline 5 & $85.50 \pm 11.65$ & $111.05 \pm 14.23$ & $78.21 \pm 13.36$ & $146.18 \pm 10.27$ & $52.67 \pm 15.81$ \\
10 & $93.92 \pm 10.04$ & $128.02 \pm 16.86$ & $86.86 \pm 6.68$ & $132.69 \pm 5.43$ & $53.89 \pm 5.58$ \\
20 & $92.91 \pm 8.70$ & $97.64 \pm 4.97$ & $90.57 \pm 9.19$ & $101.09 \pm 7.12$ & $57.11 \pm 6.45$ \\
\hline
\end{tabular}


Table 6. The abilities for five kits detecting aflatoxin $B_{1}$ in feed samples

\begin{tabular}{lccc}
\hline & Accurate number & False positive & False negative \\
\hline Kit A & 29 & 0 & 1 \\
Kit B & 25 & 4 & 1 \\
Kit C & 22 & 8 & 0 \\
Kit D & 28 & 1 & 1 \\
Kit E & 20 & 10 & 0 \\
\hline
\end{tabular}

kit D had the highest LOD. A survey evaluated the mycotoxin contamination in feed and feed raw materials worldwide using HPLC method in particular for more complex matrices which would interfere with the ELISA method, such as DDGS, finished feed, silage, or straw. Only single commodities such as maize or wheat were analysed with ELISA (Streit et al., 2013). Matrix effect is especially obvious in cases of high complexity of the test material, which can lead to overestimates, underestimates, or even false negative or false positive results (Binder, 2007). As a general rule, ELISA methods allow a certain degree of false positive rate because positive samples will be confirmed again by HPLC or other analysis methods. When the concentration of $\mathrm{AFB}_{1}$ in a sample exceeds the legal limit, all kits could detect correctly.

\section{CONCLUSION}

The present study confirmed that significant variations in sensitivity and accuracy for $\mathrm{AFB}_{1}$ detection existed among the 5 commercial ELISA kits. It can be concluded that kit A has good linear regression and correct rate; kit B has good linear regression and low correct rate; kit $\mathrm{C}$ and $\mathrm{E}$ showed low correct rate with high false positive rate, and kit D performed good accuracy under a highest LOD of them. Besides, kits products from different manufactures had great differences in CVs of intra-plate and inter-plate. Therefore, all parameters used for quality evaluation which had mentioned above should be considered comprehensively. For $\mathrm{AFB}_{1}$ detection, early studies showed that detection limits of ELISA methods are comparable to HPLC (Anklam et al., 2002) and that is one of most important reasons why ELISA kits are widely used. Although many commercial ELISA kits are now available for the detection of $\mathrm{AFB}_{1}$, their quality remarkably differs.

In conclusion, the present study showed that there are significant differences in qualities among the ELISA kits for $\mathrm{AFB}_{1}$ detection. Our data emphasized the necessity to regulate the market for $\mathrm{AFB}_{1}$ ELISA kits and reminded users to do some assessments before using in order to obtain more reliable data. It is also important for the regulatory authorities to keep monitoring these products to ensure consumer rights and feed safety.

\section{ACKNOWLEDGMENTS}

The authors are very grateful to Special Fund Project for Beijing Poultry Industry Innovation Team in Modern Agricultural Technology System (Serial number, C21108) and Special Fund for Agro-scientific Research in the Public Interest (Serial number, 20120323).

\section{REFERENCES}

Anklam, E., J. Stroka, and A. Boenke. 2002. Acceptance of analytical methods for implementation of EU legislation with a focus on mycotoxins. Food Control 13:173-183.

Anukul, N., K. Vangnai, and W. Mahakarnchanakul. 2013. Significance of regulation limits in mycotoxin contamination in Asia and risk management programs at the national level. J. Food Drug Anal. 21:227-241.

Binder, E. M. 2007. Managing the risk of mycotoxins in modern feed production. Anim. Feed Sci. Technol. 133:149-166.

Binder, E. M., L. M. Tan, L. J. Chin, J. Handl, and J. Richard. 2007. Worldwide occurrence of mycotoxins in commodities, feeds and feed ingredients. Anim. Feed Sci. Technol. 137:265282.

Bryden, W. L. 2012. Mycotoxin contamination of the feed supply chain: Implications for animal productivity and feed security. Anim. Feed Sci. Technol. 173:134-158.

Commission of the European Communities. 2006. Commission Regulation (EC) No. 401/2006 of 23 February 2006 laying down the methods of sampling and analysis for the official control of the levels of mycotoxins in foodstuffs. Off. J. EU Communities. 70:12-34.

GB 13078-2001. Hygienical standard for feeds in China. The National Feed Industry Standardization Technical Committee, Beijing, China.

GB/T 17480-2008. Determination of aflatoxin B1 in animal feeding stuffs-enzyme-linked immunosorbent assay. The National Feed Industry Standardization Technical Committee, Beijing, China.

Guan, D., P. Li, Q. Zhang, W. Zhang, D. Zhang, and J. Jiang. 2011. An ultra-sensitive monoclonal antibody-based competitive enzyme immunoassay for aflatoxin $\mathrm{M}_{1}$ in milk and infant milk products. Food Chem. 125:1359-1364.

Guo, X. D., F. Wen, N. Zheng, Q. J. Luo, H. Wang, H. Wang, S. Li, and J. Q. Wang. 2014. Development of an ultrasensitive aptasensor for the detection of aflatoxin $\mathrm{B}_{1}$. Biosens. Bioelectron. 56:340-344.

Harvey, R. B., L. F. Kubena, M. H. Elissalde, D. E. Corrier, W. E. Huff, G. E. Rottinghaus, and B. A. Clement. 1991. Cocontamination of swine diets by aflatoxin and diacetoxyscirpenol. J. Vet. Diagn. Invest. 3:155-160.

He, J., K. Y. Zhang, D. W. Chen, X. M. Ding, G. D. Feng, and X. Ao. 2013. Effects of maize naturally contaminated with aflatoxin $\mathrm{B}_{1}$ on growth performance, blood profiles and hepatic histopathology in ducks. Livest. Sci. 152:192-199.

Huang, Y., D. Han, X. Xiao, X. Zhu, Y. Yang, J. Jin, Y. Chen, and S. Xie. 2014. Effect of dietary aflatoxin B1 on growth, fecundity and tissue accumulation in gibel carp during the 
stage of gonad development. Aquaculture 428-429:236-242.

IARC (International Agency for Research on Cancer). 1993. Some naturally occurring substances: food items and constituents, heterocyclic aromatic amines and mycotoxins. IARC Monographs on Evaluation of Carcinogenic Risks to Humans. 56:397-444.

Karami-Osboo, R., M. Mirabolfathy, R. Kamran, M. ShetabBoushehri, and S. Sarkari. 2012. Aflatoxin B1 in maize harvested over 3 years in Iran. Food Control 23:271-274.

Kolosova, A. Y., W. B. Shim, Z. Y. Yang, S. A. Eremin, and D. H. Chung. 2006. Direct competitive ELISA based on a monoclonal antibody for detection of aflatoxin B1. Stabilization of ELISA kit components and application to grain samples. Anal. Bioanal. Chem. 384:286-294.

Lee, N. A., S. Wang, R. D. Allan, and I. R. Kennedy. 2004. A rapid aflatoxin B1 ELISA: development and validation with reduced matrix effects for peanuts, corn, pistachio, and soybeans. J. Agric. Food Chem. 52:2746-2755.

Li, P., Q. Zhang, and W. Zhang. 2009. Immunoassays for aflatoxins. TrAC Trends Anal. Chem. 28:1115-1126.

Liu, B. H., Y. T. Hsu, C. C. Lu, and F. Y. Yu. 2013. Detecting aflatoxin $\mathrm{B} 1$ in foods and feeds by using sensitive rapid enzyme-linked immunosorbent assay and gold nanoparticle immunochromatographic strip. Food Control. 30:184-189.

Pleadin, J., A. Vulić, N. Perši, M. Škrivanko, B. Capek, and Z. Cvetnić. 2014. Aflatoxin $B_{1}$ occurrence in maize sampled from Croatian farms and feed factories during 2013. Food Control 40:286-291

Rossi, C. N., C. R. Takabayashi, M. A. Ono, G. H. Saito, E. N. Itano, O. Kawamura, E. Y. Hirooka, and E. Y. S. Ono. 2012. Immunoassay based on monoclonal antibody for aflatoxin detection in poultry feed. Food Chem. 132:2211-2216.

Streit, E., K. Naehrer, I. Rodrigues, and G. Schatzmayr. 2013. Mycotoxin occurrence in feed and feed raw materials worldwide: long-term analysis with special focus on Europe and Asia. J. Sci. Food Agric. 93:2892-2899.

Streit, E., G. Schatzmayr, P. Tassis, E. Tzika, D. Marin, I. Taranu, C. Tabuc, A. Nicolau, I. Aprodu, O. Puel, and I. P. Oswald. 2012. Current situation of mycotoxin contamination and cooccurrence in animal feed-Focus on Europe. Toxins 4:788809.

Sweeney, M. J. and A. D. Dobson. 1998. Mycotoxin production by Aspergillus, Fusarium, and Penicillium species. Int. J. Food Microbiol. 43:141-158.
Toteja, G. S., A. Mukherjee, S. Diwakar, P. Singh, B. N. Saxena, K. K. Sinha, A. K. Sinha, N. Kumar, K. V. Nagaraja, G. Bai, C. A. Krishna, S. Vanchinathan, R. Roy, and S. Parkar. 2006. Aflatoxin $\mathrm{B}_{1}$ contamination in wheat grain samples collected from different geographical regions of India: A multicenter study. J. Food Prot. 69:1463-1467.

Van Egmond H. P. and M. A. Jonker. 2004. Worldwide regulations for mycotoxins in food and feed in 2003. Food and Agriculture Organization of the United Nations. http://www.fao.org/ docrep/007/y5499e/y5499e00.htm Accessed November 11, 2004.

Wang, Y. C., J. Wang, Y. K. Wang, and Y. X. Yan. 2011. Preparation of Monoclonal Antibodies and Development of an Indirect Competitive ELISA for Fumonisin $\mathrm{B}_{1}$ detection. J. Shanghai Jiaotong Univ. Agric. Sci. 2:012.

Xie, F., W. Lai, J. Saini, S. Shan, X. Cui, and D. Liu. 2014. Rapid pretreatment and detection of trace aflatoxin $\mathrm{B}_{1}$ in traditional soybean sauce. Food Chem. 150:99-105.

Zachariasova, M., Z. Dzuman, Z. Veprikova, K. Hajkova, M. Jiru, M. Vaclavikova, M. Pospichalova, M. Florian, and J. Hajslova. 2014. Occurrence of multiple mycotoxins in European feedingstuffs, assessment of dietary intake by farm animals. Anim. Feed Sci. Technol. 193:124-140.

Zhang, A., Y. Ma, L. Feng, Y. Wang, C. He, X. Wang, and H. Zhang. 2011. Development of a sensitive competitive indirect ELISA method for determination of ochratoxin A levels in cereals originating from Nanjing, China. Food Control. 22:1723-1728.

Zhang, H., S. Wang, and G. Fang. 2011. Applications and recent developments of multi-analyte simultaneous analysis by enzyme-linked immunosorbent assays. J. Immunol. Methods 368:1-23.

Zheng, Z., C. W. Humphrey, R. S. King, and J. L. Richard. 2005. Validation of an ELISA test kit for the detection of total aflatoxins in grain and grain products by comparison with HPLC. Mycopathologia 159:255-263.

Zinedine, A., C. Juan, J. M. Soriano, J. C. Molto, L. Idrissi, and J. Manes. 2007. Limited survey for the occurrence of aflatoxins in cereals and poultry feeds from Rabat, Morocco. Int. J. Food Microbiol. 115:124-127.

Zychowski, K. E., C. Pohlenz, T. Mays, A. Romoser, M. Hume, A. Buentello, and T. D. Phillips. 2013. The effect of NovaSil dietary supplementation on the growth and health performance of Nile tilapia (Oreochromis niloticus) fed aflatoxin-B1 contaminated feed. Aquaculture 376-379:117-123. 